\title{
Correction to: The carbonation of a lime-treated soil: experimental approach
}

\author{
Dimitri Deneele (D) - Anne Dony • Johan Colin • Gontran Herrier • \\ Didier Lesueur
}

Accepted: 18 February 2021 / Published online: 26 February 2021

(C) RILEM 2021

\section{Correction to:}

\section{Materials and structures \\ https://doi.org/10.1617/s11527-021-01617-w}

Due to an error during the proof correction process Didier Lesueur's affiliation at the time of writing has not been included in the final PDF. His complete affiliations are:

Didier Lesueur

Lhoist Southern Europe, 15 rue Henri Dagallier, 38040, Grenoble, France.

\section{Present Address:}

D. Lesueur

IMT Lille Douai, Institut Mines Télécom, Univ. Lille, Centre Materials and Processes, Villeneuve d'Ascq, France.

The original article has been corrected.

Publisher's Note Springer Nature remains neutral with regard to jurisdictional claims in published maps and institutional affiliations.

The original article can be found online at https:// doi.org/10.1617/s11527-021-01617-w.

D. Deneele $(\bowtie)$

GERS-GIE, IFSTTAR, Univ Gustave Eiffel,

44344 Bouguenais, France

e-mail: dimitri.deneele@univ-eiffel.fr

D. Deneele

CNRS, Institut des Matériaux Jean Rouxel, IMN,

Université de Nantes, 44000 Nantes, France

\section{A. Dony $\cdot$ J. Colin}

Institut de Recherche en Constructibilité, Ecole Spéciale Des Travaux Publics, Université Paris-Est, 28 Avenue du Président Wilson, 94234 Cachan Cedex, France

\author{
G. Herrier \\ Lhoist Recherche Et Développement, Rue de l'Industrie \\ 31, 1400 Nivelles, Belgium \\ Present Address: \\ D. Lesueur \\ IMT Lille Douai, Institut Mines Télécom, Univ. Lille, \\ Centre Materials and Processes, Villeneuve d'Ascq, \\ France
}

